\title{
ABUSI SESSUALI SU MINORI: ASPETTI STORICI, LEGALI E DANNI ALLO SVILUPPO DEL BAMBINO
}

\section{REVISIONE ARTICOLO}

GONÇALVES, Natamy de Almeida ${ }^{1}$, DIAS, Camila Santos²

GONÇALVES, Natamy de Almeida. DIAS, Camila Santos. Abusi sessuali su minori: aspetti storici, legali e danni allo sviluppo del bambino. Revista Científica Multidisciplinar Núcleo do Conhecimento. Anno 06, Ed. 09, Vol. 01, pp. 183-208. Settembre 2021. ISSN: 2448-0959, Link di accesso: https://www.nucleodoconhecimento.com.br/psicologia-it/aspetti-storici

\section{RIEPILOGO}

Poiché è una violenza che affligge diversi bambini e adolescenti nel mondo nel corso della storia, l'abuso sessuale sui minori richiede molta attenzione, considerando che si tratta di un fenomeno complesso e, in molti casi, difficile da identificare, essendo l'abusatore di solito una persona fidata della famiglia e che ci si aspetta che si prenda cura della vittima, come genitori biologici, patrigno, zii o amici intimi, per esempio. Inoltre, la maggior parte dei casi di abuso sessuale si verificano nella casa della vittima, dove ci si aspetta poco dal bambino e dall'adolescente che si trovano in una situazione vulnerabile. Sulla base dei fattori descritti, questa ricerca presenta la seguente domanda guida: cos'è l'abuso sessuale e quali sono i suoi impatti sullo sviluppo del bambino? Questo articolo mirava a rivedere gli aspetti storici e legali dell'abuso sessuale, esplorando le complessità delle sue definizioni e presentando i possibili effetti sulla vittima. Per questo è stata fatta una rassegna bibliografica di carattere qualitativo, descrittivo ed esplorativo, suscitando riflessioni sul tema. Si è

\footnotetext{
${ }^{1}$ Post-laurea in Neuropsicologia presso la Facoltà di Tecnologia, Scienze e Formazione - FATECE, post-laurea in Psicologia organizzativa e del lavoro presso Fondazione Hermínio Ometto e laureata in Psicologia presso la stessa istituzione.

${ }^{2}$ Consulente di orientamento. Master in Psicologia come Scienza e Professione (PUCCamp), Specialista in Educazione e Psicopedagogia (PUCCamp), Specialista in Neuropsicologia (Ospedale Ospedale Israelita Albert Einstein Institute), Laureato in Psicologia (Fondazione Hermínio Ometto - FHO).
}

RC: 98978

Disponibile in: https://www.nucleodoconhecimento.com.br/psicologia-it/aspetti-storici 
visto che il bambino e l'adolescente non avevano sempre il supporto legale, avendo subito diverse violenze senza la dovuta punizione per i loro aggressori. Nel corso del tempo, sono state istituite leggi $e$, in Brasile, lo Statuto dei bambini e degli adolescenti (ECA) aveva segnato un momento di determinazione dei diritti dell'infanzia e dell'adolescenza, stabilendo meccanismi di protezione, anche contro la violenza come l'abuso sessuale. Infine, è stato considerato in questo studio che vi è una mancanza di informazioni alla popolazione sull'argomento, che corrobora la riproduzione del suddetto reato. È stato osservato che, considerando i danni alla salute biopsicosociale della vittima e della sua famiglia, sono stati realizzati studi e progetti di prevenzione, nonché istituito diverse forme di denuncia di questo crimine. $\mathrm{Si}$ è concluso che è necessario preparare strategie per preparare le famiglie, i professionisti e le istituzioni responsabili di garantire i diritti dei bambini e degli adolescenti in modo che possano contribuire a rafforzare la lotta contro gli abusi sessuali.

Parole chiave: Abuso sessuale, Violenza sessuale su minori, Bambino e adolescente.

\section{INTRODUZIONE}

Quando si tratta di un argomento presente in diverse parti del mondo e in vari periodi storici, l'abuso sessuale rimane oggi ed è un argomento importante da affrontare, poiché tale violenza è stata continuamente riprodotta. Waiselfisz (2012) mostra che, secondo i registri del Sistema di informazione sulle malattie soggette a denuncia (SINAN), nel 201110.425 bambini vittime di abusi sessuali sono stati curati in Brasile. Nel 2018, il Brasile ha registrato almeno 32.000 casi di abusi sessuali contro bambini e adolescenti nel 2018, il più alto tasso di notifiche mai registrato dal Ministero della Salute. Dal 2011, gli agenti sanitari hanno l'obbligo di calcolare le cure. Da allora, i numeri crescono di anno in anno e si sommano a un totale di 177.300 notifiche a livello nazionale[3]. Tuttavia, come indicato da Aded et al. (2006), si ritiene che i casi identificati o riportati non dicano la realtà del numero di casi. Uno 
dei motivi è che, nella maggior parte dei casi, l'aggressore è molto vicino o parte della famiglia della vittima; un altro fattore è la mancata identificazione dei casi, o anche la convinzione che non ci sia modo di provare l'atto di abuso, intendendo come prova concreta solo la visita medica. Pertanto, si ritiene che il numero di casi sia molto più alto di quanto stimato.

Sulla base dei fattori descritti, questa ricerca presenta la seguente domanda guida: cos'è l'abuso sessuale e quali sono i suoi impatti sullo sviluppo del bambino? Questo articolo mirava a rivedere gli aspetti storici e legali dell'abuso sessuale, esplorando le complessità delle sue definizioni e presentando i possibili effetti sulla vittima.

Si tratta di una rassegna bibliografica di carattere qualitativo, descrittivo ed esplorativo, per la quale i libri sono stati utilizzati come fonte di dati, oltre agli articoli scientifici che sono stati trovati sul sito di ricerca Google. In questa ricerca sono stati utilizzati anche alcuni siti che presentano dati importanti sull'argomento.

Considerando che l'abuso sessuale è una violenza che avviene in modi che di solito ostacolano la sua identificazione, oltre a provocare molti dubbi su come affrontarlo, è necessario sviluppare ulteriori ricerche in modo che vengano stabilite sempre più conoscenze dei meccanismi che coinvolgono questo crimine e, quindi, vengano stabilite più strategie di combattimento. Pertanto, questa ricerca mostra la sua rilevanza sociale e scientifica, contribuendo al chiarimento sull'argomento e per altre ricerche da svolgere da esso.

\section{ABUSO SESSUALE: DEFINIZIONI, STORIE, LEGGI}

L'abuso sessuale è un fenomeno sociale che richiede un'attenzione raffinata perché è una questione complessa, che coinvolge diversi problemi e genera sofferenza per molti bambini, adolescenti e famiglie, che è uno dei diversi tipi di violenza. Considerando le varie definizioni di abuso sessuale, si può evidenziare quella data dal Ministero della Salute (BRASIL, 2002, p.13):

RC: 98978

Disponibile in: https://www.nucleodoconhecimento.com.br/psicologia-it/aspetti-storici 


\begin{abstract}
Abuso sexual - consiste em todo ato ou jogo sexual, relação heterossexual ou homossexual cujo agressor está em estágio de desenvolvimento psicossexual mais adiantado que a criança ou o adolescente. Tem por intenção estimulá-la sexualmente ou utilizá-la para obter satisfação sexual. Apresenta-se sobre a forma de práticas eróticas e sexuais impostas à criança ou ao adolescente pela violência física, ameaças ou indução de sua vontade. Esse fenômeno violento pode variar desde atos em que não se produz o contato sexual (voyerismo, exibicionismo, produção de fotos), até diferentes tipos de ações que incluem contato sexual sem ou com penetração. Engloba ainda a situação de exploração sexual visando lucros como é o caso da prostituição e da pornografia.
\end{abstract}

Pertanto, essendo considerato un atto di violenza, l'abuso sessuale può essere commesso in modi diversi, da quelli in cui non c'è contatto fisico tra l'aggressore e il bambino o l'adolescente, come il voyeurismo in cui si verifica l'osservazione della nudità. soddisfazione, ed esibizionismo, in cui l'aggressore mostra i propri organi sessuali. Può avvenire anche mostrando alla vittima video e riviste pornografiche, risvegliandone la sessualità in modo precoce e distorto; dare denaro, caramelle o regali al bambino o all'adolescente per consentire all'aggressore di abusare del proprio corpo; violentandola con il sesso orale, che è anche un mezzo per trasmettere malattie sessualmente trasmissibili (MST); o anche fare sesso sia vaginale che anale. Può verificarsi anche senza che la vittima se ne accorga, o fino al punto di provocare lesioni fisiche e malattie psicologiche. Inoltre, tale abuso non è commesso solo da maschi adulti sui bambini: può essere perpetrato da adolescenti, donne, anziani e da persone diverse in circostanze diverse. Questa variazione nei modi in cui può verificarsi l'abuso sessuale può rendere difficile identificare alcuni tipi che spesso non sono riconosciuti dagli adulti come tali (BRASIL, 2002).

Inoltre, come punteggiato dal Ministero della Salute (BRASIL, 2002), l'abuso sessuale comprende lo sfruttamento sessuale, che è quando coinvolge denaro, regali o qualcosa del genere, in una commercializzazione del corpo del bambino e / o dell'adolescente, come negli esempi citati, che sono la prostituzione e la pornografia. Tuttavia, come sottolineano Vega e Paludo (2015, p.48), "definire lo sfruttamento sessuale è stato un compito arduo, anche per il campo accademico". Faleiros e Campos (2000), in un'analisi del vocabolario del tema, hanno identificato 
un'inesattezza terminologica. Gli autori danno un esempio: l'abuso sessuale è stato stabilito come un crimine sessuale, vittimizzazione sessuale, abuso sessuale, violenza sessuale, violenza sessuale, sfruttamento sessuale, abuso sessuale, indignazione sessuale e lesioni sessuali. Per nominare la violenza intrafamiliare, sono stati trovati i seguenti termini: incesto, abuso sessuale incestuoso, violenza sessuale domestica, abuso sessuale domestico.

O uso sexual de menores de idade com fins lucrativos é designado ora como prostituição infanto-juvenil, ora como abuso sexual, ora como exploração sexual comercial. Por outro lado, um mesmo termo pode designar distintas realidades, como, por exemplo, o termo exploração sexual é utilizado pela OMS para designar situações de abuso sexual intra e extrafamiliar e prostituição, enquanto muitos autores o utilizam referindo-se apenas à exploração sexual comercial (FALEIROS; CAMPOS, 2000, p.4).

Pertanto, ci sono anche divergenze tra gli autori per quanto riguarda il vocabolario appropriato per ogni situazione. Tuttavia, per Faleiros e Campos (2000), I'uso di termini diversi come sinonimi e come se fossero equivalenti allo stesso concetto, non è solo terminologia, ma una questione di portata epistemologica, cioè denuncia la mancanza di concettualizzazione del problema. Questa incertezza teorica e concettuale è dovuta alla complessità e alla diversità, nonché al fatto che l'annullamento, la consapevolezza e la gestione di questo problema sono ancora recenti, così come la preoccupazione per l'infanzia, come si vedrà di seguito.

Oltre a verificarsi in modi diversi, l'abuso sessuale sui minori proviene da diversi luoghi del pianeta e descritto fin dall'antichità, come mostrato da Aded et al. (2006). Tuttavia, i progressi nella prevenzione e nell'attenuazione delle conseguenze sono stati scarsi. Ciò che può rendere difficile affrontare il problema è il fatto di passare attraverso complessità culturali, religiose, legali e professionali provenienti da tutto il mondo.

Oliveira (2006) sottolinea che nel corso della storia, una delle caratteristiche che ha contraddistinto la cultura umana è stata la negligenza contro bambini e adolescenti,

RC: 98978

Disponibile in: https://www.nucleodoconhecimento.com.br/psicologia-it/aspetti-storici 
poiché non c'era idea di fragilità come caratteristica dell'infanzia, e questo problema è stato poco discusso, così come non c'erano politiche di protezione.

L'autore presenta diversi punti della storia che esemplificano questa negligenza, denotando che in molti popoli dell'antichità, l'abuso sessuale sui minori era considerato normale e, inoltre, molti di questi popoli non distinguevano tra il bambino e l'adulto, come a Sparta, per esempio. Afferma inoltre che in Occidente, durante il Medioevo, anche questa distinzione non fu fatta, e il bambino rimase esposto a diversi tipi di punizioni; "[...] non appena non ha avuto bisogno di cure materne di base, è stata vista come adulta e quindi è cresciuta" (OLIVEIRA, 2006, p. 11). Pertanto, il bambino molto piccolo non era considerato un essere umano e il tasso di mortalità era alto, un fatto che era inteso come un fenomeno comune.

Azambuja (2006, p.3) presenta che fu solo alla fine del $17^{\circ}$ secolo che il bambino iniziò ad avere una distinzione dalla persona adulta. Fino a quel momento, le scuole non erano organizzate per età, essendo frequentate da bambini e adulti nello stesso luogo. Per l'autore, "[...] con l'emergere della comprensione che l'infanzia è una fase distinta della vita adulta, la punizione, la punizione fisica, le percosse attraverso fruste, bastoni e ferri da stiro sono usati come strumenti necessari per l'educazione" (AZAMBUJA, 2006, p.3). Nel 1780, in Inghilterra, i genitori potevano condannare i bambini all'impiccagione, sotto più di duecento tipi criminali. A New York, nel 1871, una ragazza subì gravi maltrattamenti e, poiché non c'era posto per una tale denuncia, il caso della ragazza Mary Ellen fu infine deferito alla Società per la prevenzione della crudeltà verso gli animali, in modo che potesse essere indagato dal tribunale. II caso, che ha causato molta indignazione della popolazione, è stato ciò che ha incitato la fondazione di una società incentrata sulla protezione del bambino.

Secondo Azambuja (2006), per quanto riguarda il Brasile, le prime navi lanciate in mare dal Portogallo, erano composte da uomini e bambini orfani del re, che fornivano servizi durante il viaggio e sono stati sottoposti ad abusi sessuali da parte dei

RC: 98978

Disponibile in: https://www.nucleodoconhecimento.com.br/psicologia-it/aspetti-storici 
marinai. Dice che se si verificavano tempeste, i bambini erano i primi "carichi" ad essere gettati in mare.

Guerra et al. (2013) sottolinea che fino all'inizio del XX secolo, lo Stato non si preoccupava di sviluppare politiche sociali rivolte a bambini e adolescenti. Così, la Chiesa cattolica è stata quella che ha sviluppato importanti azioni per prendersi cura dei bambini e degli adolescenti chiamati "abbandonati". Le Sante Case della Misericordia non si prendevano cura solo dei malati e dei malati, ma anche dei bambini i cui diritti erano stati violati. Così, nel tentativo di ridurre il numero di bambini concepiti al di fuori del matrimonio e che furono abbandonati anche per strada, a causa delle pressioni sociali dell'epoca, la Chiesa cattolica creò la Ruota degli Esposti. Era un dispositivo cilindrico in cui il bambino veniva collocato e lasciato, al fine di garantire l'anonimato di coloro che commettevano l'abbandono.

Alla fine della prima guerra mondiale, le organizzazioni internazionali di nuova costruzione iniziarono ad articolare i codici dei diritti umani. Nuovi organismi hanno iniziato a prestare attenzione ai diritti del bambino in particolare. La neonata Agenzia Internazionale del Lavoro (ora Organizzazione) restituì le sue convenzioni per garantire i diritti dei bambini lavoratori, così come nel 1919, alla Convenzione sul lavoro notturno degli adolescenti (industria); sempre nel 1921, la Convenzione sull'età minima (agricoltura). Tuttavia, la maggior parte della legislazione prodotta tra le due guerre mondiali non spiegava la specificità del diritto dei bambini e degli adolescenti come distinto dai diritti diretti degli adulti (UNICEF, 2009).

La prima definizione di diritti formali del bambino, che è stata creata dalle nascenti organizzazioni internazionali, ha avuto origine dal lavoro di Eglantyne Jebb che fondò in Inghilterra, nel 1919, la Save the Children, e si stabilì a Ginevra, l'anno successivo , Unione Save the Children International, per raccogliere fondi per aiuti di emergenza ai bambini che hanno sofferto a causa della prima guerra mondiale. Jebb, nel 1923, ha dichiarato la sua posizione in relazione ai diritti del bambino: "[...] credo che dobbiamo rivendicare determinati diritti per il bambino e lavorare affinché siano riconosciuti a livello internazionale" (UNICEF, 2009, p.4) . Così, I'Unione 
Internazionale Save the Children ha creato la proposta preliminare di una dichiarazione che richiedeva diritti specifici della popolazione infantile e, il 26 settembre 1924, la Società delle Nazioni l'ha adottata nella Dichiarazione di Ginevra dei diritti dell'infanzia .

Così, la Dichiarazione di Ginevra ha discusso cinque principi fondamentali: il diritto alle condizioni per lo sviluppo materiale e spirituale; il diritto di priorità in situazioni di sollievo in situazioni rischiose; diritto all'aiuto in circostanze di fame, disabilità, malattia, delinquenza o orfanotrofio; il diritto alla formazione che guida il bambino alla vita nella società; e il diritto alla protezione contro lo sfruttamento (UNICEF, 2009).

Secondo il Fondo delle Nazioni Unite per l'infanzia (UNICEF, 2009), la seconda guerra mondiale ha sostenuto la creazione delle Nazioni Unite (ONU). La fusione dell'Associazione internazionale per il benessere del bambino, con sede a Bruxelles, e dell'Unione internazionale Save the Children, ha portato all'Unione internazionale per il benessere dell'infanzia (International Union for Child Welfare - IUCW), che ha portato le Nazioni Unite ad approvare l'accordo di Ginevra Dichiarazione.

II sito Portal Brasil[4] mostra che nel 1927 il paese promulgò il primo Codice dei Minori. All'epoca, casi di grande ripercussione pubblica spinsero l'allora presidente Washington Luís a stabilire che 18 anni fosse l'età minima per arrestare i trasgressori. Quello che è successo con il ragazzo di 12 anni Bernardino è stato uno di quei casi. Nel 1926, il ragazzo, che era un lustrascarpe, si arrabbiò con un cliente a causa del suo rifiuto di pagare il servizio fornito per le strade di Rio de Janeiro. II bambino avrebbe lanciato vernice a questo ragazzo e così è finito in prigione per quattro settimane. Bernardino è stato violentato nella sua cella da 20 adulti, cosa che ha provocato l'indignazione dei medici quando il ragazzo si è recato a Santa Casa.

Così, la diffusione del caso causò una forte controversia, avviando una discussione pubblica che raggiunse le ruote del Congresso, così come il Palazzo Catete, dove era il luogo del governo federale. Così il presidente ha scelto il 12 ottobre (Giornata dei bambini) per firmare il Codice dei minori, che è stata la prima legislazione nel 
paese per l'infanzia e l'adolescenza in particolare. Un articolo di questo codice vietava la Ruota degli Esposti. "Con il codice, la madre dovrebbe registrare il bambino e quindi consegnarlo in un orfanotrofio" (BRASIL, s/d).

Il sito web della Dichiarazione Universale dei Diritti Umani[5] (UDHR) mostra che il 10 dicembre 1948, a Parigi, tale dichiarazione è stata proclamata dall'Assemblea Generale delle nazioni, attraverso la Risoluzione 217 A (III). È un documento che ha segnato la storia dei diritti umani. La UDHR è stata sviluppata da diversi rappresentanti di vari background legali e culturali di tutto il mondo, ed è quindi una norma comune in modo che raggiunga tutti i popoli e le nazioni. Questa affermazione viene con l'opinione che l'uomo ha diritto a una vita dignitosa: "Mentre il riconoscimento della dignità inerente a tutti i membri della famiglia umana e dei loro diritti uguali e inalienabili è il fondamento della libertà, della giustizia e della pace nel mondo [...]" (UDHR, 1948).

In questa occasione, I'ONU era più impegnata nel discutere i principi dell'UDHR, ma la stessa IUCW ha difeso l'idea di un nuovo documento, che fosse specifico per i diritti del bambino, aggiornando la precedente dichiarazione e ribadendo il principio che il bambino dovrebbe ricevere ciò che l'umanità ha di meglio da offrire. La Dichiarazione dei diritti dell'infanzia è stata adottata dall'ONU solo il 20 novembre 1959. L'approvazione dell'Assemblea generale è stata importante poiché ha evidenziato la necessità di considerare separatamente i diritti dell'infanzia. La Dichiarazione dei diritti del fanciullo ha evidenziato il benessere emotivo dei bambini e ha garantito il loro diritto ad avere la priorità nel ricevere protezione in caso di emergenza. "Nonostante questi cambiamenti, il documento del 1959 ha mantenuto un approccio assistenzialista, mirando a salvare, custodire e proteggere il bambino, dando poca importanza all'aumento del loro potere" (UNICEF, 2009, p.5).

Inoue e Ristum (2008) denotano che la violenza è stata la principale causa di morte principalmente nel pubblico giovane e infantile. Negli Stati Uniti, non è stato fino al 1960 che la violenza contro i bambini è stata vista come un problema medicosociale. "soprattutto [...] i pediatri hanno iniziato ad affrontare il tema che mostra le 
sequele del maltrattamento e le discrepanze tra le prove radiologiche sulle lesioni e le spiegazioni degli aggressori" (MINAYO, 2002, p. 109). Sebbene questo movimento all'interno della medicina abbia provocato critiche e accuse di "medicalizzazione della violenza", ha avuto una grande influenza sulla produzione teorica, innescando la ricerca, nonché sui movimenti a favore della difesa dei diritti dei bambini. Così, il campo medico iniziò una repulsione sociale sugli abusi sessuali, che prese consistenza anche dagli Stati Uniti, oltre a rafforzare il movimento femminista del 1970.

L'anno 1973 è stato segnato da un evento che ha reso il 18 maggio, un giorno importante nel calendario del Ministero della Salute, memorado ai giorni nostri. II fatto è che, in questa data, Araceli Cabrera Crespo, di nove anni, è scomparsa dalla scuola in cui studiava, non essendo più vista viva. II sito web del National Health Council spiega che "Araceli è stato picchiato, violentato, drogato e ucciso in un'orgia di droghe e sesso. II suo corpo, il suo viso era principalmente sfigurato di acido" (BRASIL, s/d). Sei giorni dopo l'incidente, il corpo è stato trovato in un lotto vuoto vicino al centro della città di Vitória, Espírito Santo. Tale martirio ha avuto un grande significato, quindi questa data è considerata e ricordata come la Giornata nazionale per combattere l'abuso sessuale e lo sfruttamento di bambini e adolescenti.

Tra gli anni '60 e '70 la mobilitazione a favore dei diritti dei bambini e degli adolescenti è stata portata avanti da organizzazioni non governative (ONG), che hanno fatto grandi passi avanti; in questo modo, queste organizzazioni hanno sollecitato le Nazioni Unite a dichiarare l'anno 1979 come Anno Internazionale del Bambino, nella proposta di richiamare l'attenzione sulle questioni che riguardano l'infanzia. "Una volta stabilito questo accordo, il governo della Polonia ha presentato alla Commissione delle Nazioni Unite sui diritti umani un testo preliminare di una convenzione sui diritti dell'infanzia" (UNICEF, 2009, p.5). Pertanto, era evidente che la finalizzazione di questo documento avrebbe richiesto una migliore preparazione e più tempo. II processo è quindi durato un decennio, considerando che la stesura di

RC: 98978

Disponibile in: https://www.nucleodoconhecimento.com.br/psicologia-it/aspetti-storici 
un trattato che coinvolga diversi ambiti dell'interpretazione culturale e sociale è un lavoro che richiede cautela.

Minayo (2002) dice che nel 1980, nel campo della salute pubblica mondiale, la violenza si presenta come una questione sostanziale. I movimenti di assistenza specializzata e prevenzione nascono dal riconoscimento della morbilità e della mortalità attraverso la violenza, come un grave problema per la salute nei suoi aspetti sociali, nonché il bersaglio dell'assistenza primaria, secondaria e terziaria. Sempre nel 1980, in Brasile, si cominciarono a pensare proposte, al ritmo del movimento sociale che confermava la Costituzione del 1988. Solo quest'anno il bambino è considerato un tema di diritti, grazie alla Costituzione federale, e avrà la Convenzione delle Nazioni Unite, in una nuova era di difesa dei diritti dei bambini e degli adolescenti.

Tre anni prima di questa costituzione, il 4 luglio 1985, il Centro Regionale per l'Attenzione agli Abusi sui Minori (CRAMI), è stato fondato da un gruppo composto da diversi professionisti appartenenti alla Pontificia Università Cattolica di Campinas (PUC-Campinas), coinvolgendo rappresentanti di vari settori della comunità, come l'Ordine degli Avvocati Brasiliani (OAB) - Campinas, Tribunale dei Minori, tra gli altri. Secondo il sito web di CRAMI[6], "Preoccupato per i normali casi di bambini che arrivavano negli enti pubblici, mostrando segni di percosse, ustioni, abrasioni, emorragie interne, questo gruppo ha fondato CRAMI". Molti di questi bambini sono morti e i tutori lo hanno spiegato come una caduta o una mancanza di attenzione da parte del bambino. Nel tempo è stato creato un protocollo per la gestione dei casi di violenza domestica. Dall'accertamento del caso, sono stati raccolti i dati e l'assistente sociale ha proceduto all'assistenza nelle case delle vittime, con l'obiettivo di avere un contatto con la situazione reale e, a seconda della gravità del caso, deferire la vittima al Tribunale dell'Infanzia.

II sito web CRAMI informa inoltre che "il lavoro sviluppato dai professionisti altamente qualificati di CRAMI ha iniziato a diventare un riferimento e ha incoraggiato l'apertura di altri CRAMI nello stato di San Paolo". Con la determinazione del Sistema Unificato

RC: 98978

Disponibile in: https://www.nucleodoconhecimento.com.br/psicologia-it/aspetti-storici 
di Assistenza Sociale (SUAS), nel 2014 CRAMI ha esteso la propria assistenza a tutte le persone in situazioni di vulnerabilità e rischio sociale, non essendo più limitata alla cura di bambini e adolescenti vittime di violenza domestica. È un'entità non governativa, senza scopo di lucro e ha l'assistenza di psicologi, assistenti sociali ed educatori sociali, che segue la missione, secondo il sito web, di "Costruire un futuro migliore per bambini, adolescenti e vittime vulnerabili della violenza domestica".

II 20 novembre 1989, l'Assemblea Generale delle Nazioni Unite ha adottato la Convenzione sui diritti del fanciullo, entrando in vigore il 2 settembre 1990. Questo è il più completo di tutti i trattati sui diritti umani e gli strumenti giuridici per la protezione dei diritti dei minori. Sebbene esistano disposizioni in difesa dei diritti del fanciullo nelle attrezzature internazionali per i diritti umani, la Convenzione è la prima a coprire e articolare tutti gli aspetti rilevanti dei diritti importanti per il bambino, essendo economici, culturali, politici e sociali. Tuttavia, è stata la prima attrezzatura internazionale che ha esplicitamente riconosciuto che il bambino è un attore sociale in possesso dei propri diritti (UNICEF, 2009).

Secondo I'UNICEF (2009, p. 2), "Per mezzo delle disposizioni del trattato, gli Stati Parti sono tenuti per legge a soddisfare i diritti di tutti i bambini". La Convenzione prevede 54 articoli e si basa su quattro principi fondamentali: l'interesse superiore del minore; non discriminazione; rispetto per le opinioni del bambino; e il diritto alla sopravvivenza e allo sviluppo. L'obiettivo generale che ha e l'importanza che dà alla rappresentazione che favorisce il bambino determinano l'incessante pertinenza di tutte le azioni volte a soddisfare i diritti del bambino. "La Convenzione riafferma e arricchisce i diritti umani in modo significativo" (UNICEF, 2009, p. 2). Li ribadisce applicando direttamente al bambino molti dei principi essenziali dei documenti internazionali sui diritti umani preesistenti, come la non discriminazione e l'universalità; li rafforza istituendo ed espandendo i dispositivi inclusi in altre attrezzature per i diritti umani, chiarendo le responsabilità degli Stati parti in relazione al bambino. Integra i diritti dei bambini che non sono stati incorporati ampiamente.

RC: 98978

Disponibile in: https://www.nucleodoconhecimento.com.br/psicologia-it/aspetti-storici 
Sottolinea che la responsabilità per la realizzazione dei diritti del bambino dovrebbe essere attribuita a coloro che hanno doveri ad esso correlati, come la famiglia e le persone responsabili del bambino e gli Stati parti.

La convenzione ha un significato che va oltre le sue implicazioni giuridiche. Lo strumento ha anche contribuito a una trasformazione degli atteggiamenti nei confronti dell'infanzia. In dever, la Convenzione ha determinato i termini dell'infanzia, stabilendo gli standard minimi per la cura, il trattamento, la sopravvivenza, la protezione, la partecipazione e lo sviluppo, che sono i diritti di tutti gli individui la cui età è inferiore ai 18 anni di età. I suoi articoli riaffermano che è necessario proteggere l'infanzia come un diverso periodo dell'età adulta e stabilire un tempo durante il quale il bambino cresce, impara, gioca e si sviluppa, in modo che poi i diritti del bambino siano pienamente realizzati (UNICEF, 2009).

Come mostra Lemos (2008), inaugurando una nuova prospettiva di diritti e doveri basata sulla dottrina della protezione integrale, lo Statuto dei bambini e degli adolescenti (ECA), è stato promulgato nel 1990, come legge federale n. 8.069/90, garantendo al bambino e all'adolescente il rispetto come persone in particolare sviluppo:

Art. 3o A criança e o adolescente gozam de todos os direitos fundamentais inerentes à pessoa humana, sem prejuízo da proteção integral de que trata esta Lei, assegurando-se Ihes, por lei ou por outros meios, todas as oportunidades e facilidades, a fim de lhes facultar o desenvolvimento físico, mental, moral, espiritual e social, em condições de liberdade e de dignidade (BRASIL, 1990).

Questa legge stabilisce un nuovo diagramma, stabilendo pratiche diverse in alcuni aspetti di quelle previste dal Codice dei Minori del 1927 e del 1979. "Se, nel Codice dei Minori, l'oggetto era il più piccolo; nello Statuto dei bambini e degli adolescenti, l'oggetto è il bambino e l'adolescente come soggetti di diritti" (LEMOS, 2008, p.98). In questo modo, viene inaugurata una nuova identità sociale chiamata bambini e adolescenti. Non sono solo le pratiche che sono cambiate, così come l'oggetto è un altro.

RC: 98978

Disponibile in: https://www.nucleodoconhecimento.com.br/psicologia-it/aspetti-storici 
La Corte dei conti europea presenta una proposta per costruire un modello di protezione integrale per i bambini e gli adolescenti, non solo essendo limitato all'attenzione dopo che i diritti sono stati violati, ma prima che la violazione avvenga, come previsto dal suo art.70: "È dovere di tutti prevenire il verificarsi di una minaccia o di una violazione dei diritti dei bambini e degli adolescenti" (BRASIL, 1990). Questa legge federale garantisce, tra gli altri aspetti, la protezione del bambino contro qualsiasi tipo di violenza, compresa la violenza sessuale.

È noto che, nella maggior parte dei casi, l'aggressore è vicino alla famiglia. Come dimostrato da Santos, Costa e Granjeiro (2009), la maggior parte dei casi di abuso sessuale avviene allinterno della famiglia, e sono commessi in primo luogo dai genitori biologici delle vittime e, in secondo luogo, dai patrigni. Questo è un esempio di violenza intrafamiliare che, secondo Araújo (2002), è la violenza che avviene in famiglia, coinvolgendo persone che vivono o meno nello stesso luogo, ma che non si limita alla sola famiglia, ma coinvolge soggetti in comune convivenza nello stesso spazio domestico, con o senza legami di parentela. Per questi casi, la ECA ha delle specificità e una di queste è menzionata nell'articolo 101:

$\S 2^{\circ}$ Sem prejuízo da tomada de medidas emergenciais para proteção de vítimas de violência ou abuso sexual e das providências a que alude 0 art. 130 desta Lei, o afastamento da criança ou adolescente do convívio familiar é de competência exclusiva da autoridade judiciária e importará na deflagração, a pedido do Ministério Público ou de quem tenha legítimo interesse, de procedimento judicial contencioso, no qual se garanta aos pais ou ao responsável legal o exercício do contraditório e da ampla defesa (BRASIL, 1990).

Queste leggi per proteggere i bambini e gli adolescenti dagli abusi sessuali sono estremamente necessarie, poiché si occupano di qualcosa di grave e possono causare molti danni alla vittima, come verrà sottolineato di seguito.

\subsection{DANNI ALLA VITTIMA E POSSIBILI AZIONI}

Tra le conseguenze che l'abuso sessuale può causare a bambini e adolescenti, si possono evidenziare: disturbi cognitivi, comportamentali, emotivi e sociali; 
isolamento sociale, paura esagerata, difficoltà di adattamento, idee omicide e suicide, apprendimento del deficit linguistico, perdita di interesse per giochi e studi, fughe da casa, automutilazione, isolamento sociale, aggressività e altre conseguenze che possono compromettere seriamente la vita della vittima (SERAFIM et al., 2011).

Prado e Carneiro (2005) discutono, dal punto di vista della psicoanalisi, del trauma che, secondo loro, il termine enfatizzerebbe il danno generato nella capacità di simboleggiare e trasformare, nonché la conferma delle zone psichiche morte, che interferiranno nelle generazioni future, raggiungendo le loro scelte amorose e la loro prospettiva di raggiungere la loro sessualità.Gli autori discutono da due tipi di traumi, proponendo di chiamarli attivi e cumulativi, e il primo rimane nella psiche della persona ripetutamente, soprattutto quando si tratta di abusi sessuali su minori, e può avere le varie manifestazioni, come promiscuità, prestazioni, condizioni psicopatiche, depressioni, condizioni psicosomatiche, ecc.

Il trauma cumulativo si riferisce alla tensione ricorrente di inadempimento del ruolo della madre come scudo protettivo, che lascia nella fase di sviluppo di cui il bambino ha bisogno della madre esercitando il suo ruolo; questi fallimenti materni possono essere suscettibili di correzione in base al processo di maturazione. Per quanto riguarda il trauma attivo, "allo stesso modo del trauma cumulativo, è infido, solo che invece di agire e stabilirsi durante l'infanzia e l'adolescenza, lo fa per tutta la vita, e oltre, poiché viene ristampato attraverso le generazioni" (PRADO; CARNEIRO, 2005, p.31). Pertanto, il trauma può interferire e causare sofferenza per tutta la vita della vittima, portando diverse possibilità di effetti gravi a lungo termine.

La relazione del bambino con adulti violenti, con conseguente rottura della fiducia e dell'insicurezza, favorisce la vittima a non credere nell'affidabilità e nella sicurezza delle persone in generale, che possono riflettere sulle loro relazioni future. Così, fin dall'adolescenza, ci sono casi di vittime che, nella disperazione di ottenere affetto, finiscono per diventare promiscue; d'altra parte, gli altri raramente si rendono

RC: 98978

Disponibile in: https://www.nucleodoconhecimento.com.br/psicologia-it/aspetti-storici 
disponibili per le relazioni; entrambi i casi si muovono verso l'isolamento e la depressione (PRADO; CARNEIRO, 2005).

Padilha (2002) parla della peculiarità dell'abuso sessuale, sottolineando che spesso non ci sono prove fisiche della sua consumazione e quindi l'aggressore sostiene di non aver costretto il bambino a fare nulla. "Considerare questa caratteristica del fenomeno sembra essere fondamentale nella pianificazione di interventi per la prevenzione $\mathrm{O}$ il trattamento di situazioni di abuso sessuale, intrafamiliare o extrafamiliare" (PADILHA, 2002, p. 210). Pertanto, la prevenzione di tale violenza richiede una conoscenza dei movimenti che permeano le aggressioni.

Come già accennato, il Ministero della Salute sottolinea che l'abuso sessuale "si presenta sotto forma di pratiche erotiche e sessuali imposte a bambini o adolescenti per violenza fisica, minacce [...]" ma complete: "[...] o induzione della loro volontà" (BRASIL, 2002, p.13). Quindi, in effetti, la maggior parte dei casi di abuso sessuale si verificano sotto minacce da parte dell'aggressore, all'ombra di violenza, ricatto, ecc., Tuttavia, indurre la volontà della vittima costituisce anche aggressione.

Esiste ancora attualmente un concetto errato che considera che se il bambino e soprattutto l'adolescente sono conniventi con l'atto sessuale, la situazione non costituirebbe un abuso. Tuttavia, la legge brasiliana non lascia dubbi sul fatto che i bambini e gli adolescenti siano vulnerabili poiché, come già portato a questa ricerca, se la persona si trova in una fase di sviluppo psicosessuale prima della vittima, sarà abuso sessuale (BRASIL, 2002).

Pertanto, anche se il bambino o l'adolescente acconsente all'atto sessuale, questo è chiaramente riprovevole dalla legislazione brasiliana. Affrontando i crimini sessuali contro i vulnerabili, l'articolo 217-A del codice penale chiarisce che "Avere una congiunzione carnale o praticare un altro atto libidinoso sotto i 14 (quattordici) anni: Penna - reclusione, da 8 (otto) a 15 (quindici) anni" (HARMS, 2016, p.577).

RC: 98978

Disponibile in: https://www.nucleodoconhecimento.com.br/psicologia-it/aspetti-storici 
Inoltre, il 6 novembre 2017 la Corte Superiore di Giustizia (STJ) ha lanciato il sommario 593, che determina enfaticamente che è irrilevante per la caratterizzazione del reato il consenso o meno della vittima:

O crime de estupro de vulnerável se configura com a conjunção carnal ou prática de ato libidinoso com menor de 14 anos, sendo irrelevante eventual consentimento da vítima para a prática do ato, sua experiência sexual anterior ou existência de relacionamento amoroso com o agente. (Súmula 593, TERCEIRA SEÇÃO, julgado em 25/10/2017, DJe 06/11/2017) (BRASIL, 2017).

Pertanto, la legislazione brasiliana rende ancora più chiaro che, indipendentemente dalle circostanze di accettazione o meno del bambino o dell'adolescente, i fattori menzionati sull'abuso sessuale si applicano e sono costituiti come crimini.

Inoltre, il fatto che la vittima acconsenta in qualche modo può causare un aumento del senso di colpa e aggravare il danno emotivo, oltre a corroborare il suo silenzio sulla violenza vissuta e, in molti casi, favorire il bambino o l'adolescente a continuare ad essere abusato sessualmente. Per Padilha (2002), la durata dell'abuso può consistere in un episodio isolato o di routine della durata di diversi anni senza divulgazione a terzi. L'aggressività può essere fatta con vittime di qualsiasi età, dai neonati o bambini molto piccoli, all'adolescenza.

Inoltre, le ipotesi sul comportamento di collusione con tale violenza sono stabilite in base al comportamento della madre, del padre o del patrigno, o del bambino, provenienti dalla storia della vita, che a volte ripetono schemi delle generazioni passate. La mancanza di un repertorio di autoprotezione può essere dovuta a modelli inadeguati di genitori, che potrebbero non aver avuto un modello adeguato dei loro genitori, e così via. "Questo meccanismo di ripetizione intergenerazionale dei comportamenti di esposizione al rischio spiegherebbe perché madri, padri o patrigno diventano "ciechi" in relazione all'abuso sessuale [...]" (PADILHA, 2002, p.211). Cioè, non possono identificare le prove della violenza.

Inoltre, lo stadio di sviluppo in cui si trova la vittima ha interferenze sia nel processo di scoperta che nel processo del sospetto: "L'abuso sui minori è un crimine che 
spesso può essere dimostrato solo dalla testimonianza di bambini in età prescolare. Se la testimonianza di un bambino non è corretta, un adulto innocente può essere punito ingiustamente" (PAPALIA; OLDS; FELDMAN, 2006, p. 301). Come giustamente sottolineano gli autori, il bambino può finire per "ricordare" eventi che non si sono verificati; tendono ad essere allusivi e il loro discorso può essere indotto da un'altra persona, ed è difficile discriminare la falsità del discorso di un bambino che viene intervistato da qualcuno che fa domande con dettagli di parte. Ciò può verificarsi principalmente con i bambini sotto i quattro anni di età, perché in questa fase i bambini hanno maggiori probabilità di commettere errori nel ricordare i dettagli di un evento che varia con la ripetizione. Tuttavia, Habigzang, Ramos e Ekoller (2011) si riferiscono al momento della rivelazione dell'abuso sessuale come cruciale nella vita della vittima, poiché può causare rivittimizzazionese gli adulti non credono nel linguaggio del bambino e non adottano misure protettive appropriate, che dovrebbero avere una rete affettiva e di supporto sociale, che è composta da sistemi o persone significative per la vittima.

\begin{abstract}
Nos casos de abuso sexual contra crianças e adolescentes compreende-se como constituinte da rede: família, escola, comunidade, Conselho Tutelar, Delegacia, Conselho de Direitos da Criança, Ministério Público e Juizado da Infância e Adolescência, abrigos, serviços de saúde (postos de saúde e hospitais) e assistência social (Centro de Referência da Assistência Social e Centro de Referência Especializado da Assistência Social) (HABIGZANG; RAMOS; KOLLER, 2011, p. 468).
\end{abstract}

Pertanto, tutte queste istituzioni hanno la responsabilità di proteggere la vittima, sforzandosi di pianificare e realizzare interventi che minimizzino efficacemente gli effetti della violenza subita da bambini o adolescenti (HABIGZANG; RAMOS; KOLLER, 2011). La scuola si distingue perché è un ambiente presente in fasi molto importanti della vita di bambini e adolescenti nel suo sviluppo.

Ancora chiarendo sulle implicazioni nello sviluppo della vittima di abusi sessuali, Papalia; Olds e Feldman (2006) mostrano le citazioni più comuni in base all'età: in età prescolare spiccano ansia, incubi e comportamenti sessuali inappropriati; nei bambini in età scolare si osservano comportamenti aggressivi, problemi scolastici, 
paura, iperattività e persino malattie mentali; infine, gli adolescenti presentano comunemente depressione, disturbi fisici, fuga, azioni illegali, comportamenti autodistruttivi o suicidi e abuso di sostanze.

Santos; Costa e Granjeiro (2009) sottolineano che la maggior parte dei casi di abuso sessuale si verificano all'interno della famiglia, e i casi più comuni di abuso sessuale sono praticati dai genitori biologici delle vittime e, in secondo luogo, dai patrigno. È un tipo di violenza intrafamiliare che, secondo Araújo (2002), avviene in famiglia coinvolgendo persone che risiedono o meno nello stesso luogo, ma che non è limitata solo alla famiglia, ma coinvolge soggetti di convivialità comune nello stesso spazio domestico, indipendentemente dal fatto che abbiano o meno legami di parentela. Quindi, si può affermare che c'è il rischio che le persone idealizzino un certo stereotipo per tali abusatori, non pagando per vedere che, anche se sono lavoratori, "genitori di famiglia", contribuenti, hanno una buona vita sociale, sono persone ricche, gentili e istruite o che apparentemente educano e si prendono cura dei loro figli, questo non significa che tali persone non possano commettere abusi sessuali, anche se hanno una condotta encomiabile nei confronti della società.

"La maggior parte dei pedofili non ha precedenti penali e le persone nella loro vita li descrivono come un lavoratore, religioso e zelante caregiver della loro famiglia" (COHEN; MANNARINO, 2000a; apud HABIGZANG et al., 2005, p.346).

Inoltre, gli aggressori con stereotipi visti socialmente come buoni cittadini possono provocare l'accomodamento delle persone a non preoccuparsi della prevenzione con bambini e adolescenti per quanto riguarda gli abusi sessuali, in quanto può anche confondere nel processo di sospetto e identificazione di questa violenza. Inoltre, a seconda del rapporto che l'aggressore ha con la famiglia, molti dei casi non vengono segnalati. Ad esempio, una madre non può denunciare il coniuge perché si fida di lui, non dando credito alla denuncia del bambino o dell'adolescente perché non vuole allontanarsi dal coniuge o perché dipende dall'aggressore per sostenere la famiglia, tra le altre situazioni. Williams (2002) cita un altro aspetto importante, che è il fatto che la violenza sessuale è ancora attualmente considerata tabù, e spesso la vittima 
stessa è stigmatizzata e spesso ha un senso di colpa o vergogna; questo, tra gli altri, è uno dei motivi della mancata segnalazione da parte di persone che fanno o non fanno parte della famiglia della vittima.

Tuttavia, Habigzang et al. (2005) presenta la mappatura dei fattori di rischio per l'abuso sessuale intrafamiliare che sono stati identificati nei processi legali della Procura della Repubblica di Rio Grande do Sul - Brasile, analizzando 71 file che presentano il profilo degli aggressori; una delle caratteristiche sorprendenti era che la maggior parte degli aggressori erano disoccupati o con un eventuale lavoro. "La disoccupazione è un fattore di rischio per la violenza intrafamiliare, poiché può generare stress e conflitti tra i membri della famiglia" (HABIGZANG et al., 2005, p. 345). Inoltre, il caregiver finisce per essere responsabile del bambino 0 dell'adolescente per la maggior parte del tempo, il che offre maggiori opportunità per il verificarsi di abusi sessuali. Altre caratteristiche riscontrate nella maggior parte degli abusatori di questo studio sono: basso livello di istruzione, sposato o vivente coniugalmente, consumo di alcol o droghe, tra le altre cose. È importante notare che queste caratteristiche sono state trovate in una percentuale dei casi studiati e, sebbene siano abbastanza comuni, ci sono molti abusatori che non presentano nessuna di queste caratteristiche. Pertanto, è importante che gli adulti siano attenti e cerchino informazioni che aumentino le loro conoscenze sull'argomento e quindi siano in grado di creare una rete di protezione per bambini e adolescenti, essendo in grado non solo di identificare, ma prevenire, prevenire l'abuso sessuale.

Per Brino e Williams (2005), l'abuso sessuale sui minori può essere prevenuto attraverso azioni che possono essere suddivise in tre livelli di prevenzione. II primo livello è il livello primario, che mira a eliminare o ridurre i fattori sociali, ambientali e culturali che favoriscono atti di aggressione. Pertanto, possono essere promosse azioni volte a formare professionisti e altre persone direttamente coinvolte con il bambino, come gli insegnanti. Inoltre, possono essere promosse azioni con la popolazione a rischio, promuovendo l'educazione dei bambini sui rischi di abuso sessuale.

RC: 98978

Disponibile in: https://www.nucleodoconhecimento.com.br/psicologia-it/aspetti-storici 
Santos e Ippolito (2009) sostengono che l'educazione sessuale è il modo migliore per prevenire, e che nonostante appartenga all'adulto responsabile dell'educazione di bambini e adolescenti, la responsabilità di proteggerli dagli abusi sessuali, un programma di formazione continua è in grado di preparare bambini e adolescenti a difendersi da questa violenza.

Un altro mezzo di prevenzione menzionato dagli autori è l'inclusione sociale del bambino considerato "diverso", o rifiutato dai gruppi. "Dopo la sottomissione del bambino agli abusi sessuali subiti a casa o nel quartiere o il suo silenzio davanti a lui, di solito c'è una ricerca di accettazione e affetto di una persona cara" (SANTOS; IPPOLITO, 2009, p.128). Così, molti dei bambini che sono stati vittime di abusi sessuali avevano un basso livello di autostima, e generalmente sono cresciuti in isolamento nelle proprie case e nella comunità in cui vivono, vedendosi come diversi dagli altri e non avendo orientamenti sessuali fondamentali.

Puntando alla prevenzione, Santos e Ippolito (2009) suggeriscono alle scuole di promuovere la sensibilizzazione dei familiari responsabili dell'educazione dei bambini e degli adolescenti, utilizzando la creatività dei loro educatori per lavorare con queste famiglie, incoraggiandole e insegnando loro a rafforzare i bambini e gli adolescenti contro gli abusi sessuali; mantenere un rapporto di fiducia con il bambino; dedicare tempo ad esso, e ascoltare apertamente ciò che il bambino ha da dire, non banalizzando nei casi in cui il bambino abusato "rompe il silenzio", pensando che deve inventare i fatti e; costruire una rete di sostegno sociale tra i membri della famiglia al lavoro per proteggere il bambino nei momenti in cui il membro della famiglia che è responsabile dell'educazione dei più piccoli deve lasciare la casa. Gli autori affermano che: "La maggior parte degli atti di abuso intra ed extra-familiare si verificano quando il bambino è solo con giovani e adulti nella propria casa o nella casa di conoscenti" (SANTOS; IPPOLITO, 2009, p.129).

Il secondo approccio, secondo Brino e Willians (2005), è il secondario, che mira a rilevare precocemente $\mathrm{i}$ bambini a rischio, prevenendo così il ripetersi di atti di violenza, lavorando per ridurre lo stress causato dal processo nel sistema legale che 
il bambino attraversa, considerando che la postura dei professionisti che lavorano con il bambino può ammorbidirsi o finire per produrre danni psicologici alla vittima. II terzo e ultimo approccio presentato dagli autori è quello terziario; mira a monitorare la vittima e l'aggressore, da parte di professionisti qualificati, al fine di prevenire o mitigare le perdite causate da abusi sessuali, nonché possibili sequele a lungo termine.

Considerando l'importanza di lavorare con la prevenzione degli abusi sessuali, agendo insieme ai responsabili e/o con il bambino, è possibile pianificare interventi finalizzati alla prevenzione primaria e secondaria. Le madri, ad esempio, possono svolgere un ruolo decisivo nella protezione contro questa violenza. "Quando non sono in grado di riconoscere il verificarsi di abusi sessuali e protezione, il bambino può diventare più vulnerabile agli abusi" (BRINO; WILLIANS, 2005, p.176). Da qui l'importanza di cercare modi per informare le madri sugli abusi sessuali in modo che diventino in grado di proteggere il bambino dal verificarsi di questo tipo di aggressione, e la scuola può essere un luogo strategico per tale azione, poiché è un'istituzione in grado di promuovere interventi sfruttando il contatto diretto con i genitori, bambini e adolescenti.

Brino e Williams (2005) rafforzano l'idea che consentire ai bambini e alle madri di riconoscere i segni che l'abuso sessuale sta accadendo può essere un'azione che ferma efficacemente il verificarsi di tale violenza. Questo intervento diretto al bambino includerebbe il riconoscimento dei segni di avvicinamento di un aggressore, nonché il riconoscimento di comportamenti sessuali inappropriati. Per le madri, l'azione comporterebbe il riconoscimento di comportamenti che sono indicatori di abusi di successo. Quando la proposta dell'azione è l'individuazione di bambini e adolescenti a rischio, rompendo con atti violenti o loro ripetizioni, intervenendo in casi già confermati e lavorando nella prevenzione di possibili sequele, questa prospettiva si adatta alla prevenzione secondaria o addirittura terziaria.

Oltre alla prevenzione, è di fondamentale importanza sapere cosa fare se l'abuso sessuale si è già verificato. È necessario saper denunciare, prendersi cura della 
vittima dando il supporto necessario e facendo riferimento a trattamenti specializzati (BRINO; WILLIAMS, 2003b, p.2).

Uno dei modi per riferire, come mostrato sul sito web dell'UNICEF[7], è contattare il Consiglio di Tutela, considerando che è responsabilità dei consulenti garantire i diritti dei bambini e degli adolescenti. Quando si riceve la notifica, spetta a questi professionisti analizzare l'origine di ciascun caso, effettuando visite alla famiglia. Se ciò è confermato, il Consiglio deferiste la situazione alla Procura della Repubblica.

Un altro mezzo di denuncia indicato dall'UNICEF è contattare i tribunali per l'infanzia e la gioventù, nel caso di comuni che non hanno il Consiglio di tutela. I reclami possono anche essere presentati presso le stazioni di polizia per la protezione dei bambini e degli adolescenti e le stazioni di polizia delle donne. C'è anche un'applicazione per smartphone e tablet chiamata Proteja Brasil, che mostra i telefoni e la posizione dell'istituzione specializzata più vicina a quella dell'informatore. Inoltre l'applicazione offre assistenza in caso di dubbi sul tipo di violenza.

II numero 100 - Diritti Umani è un canale in cui "i reclami possono essere anonimi o, quando richiesto dall'informatore, è garantita la riservatezza della fonte delle informazioni" (BRASIL, s/d). In questo modo chiunque può segnalarlo senza essere identificato. Denunciare non è sinonimo di accusare, poiché è il professionista specializzato che indagherà sui fatti. Pertanto, è importante sottolineare che non è necessario essere sicuri che si sia verificato un abuso sessuale per la denuncia. È un atto di impegno per la sicurezza e il benessere del bambino e dell'adolescente, e l'omissione è un atto grave quanto l'abuso sessuale stesso. La ECA, in un unico paragrafo dell'articolo 70-B, stabilisce che

São igualmente responsáveis pela comunicação de que trata este artigo, as pessoas encarregadas, por razão de cargo, função, ofício, ministério, profissão ou ocupação, do cuidado, assistência ou guarda de crianças e adolescentes, punível, na forma deste Estatuto, o injustificado retardamento ou omissão, culposos ou dolosos (BRASIL, 1990).

RC: 98978

Disponibile in: https://www.nucleodoconhecimento.com.br/psicologia-it/aspetti-storici 
Lo Statuto, poi, sottolinea la responsabilità delle persone, che sono in qualche modo coinvolte nella vita del bambino e dell'adolescente, di presentare la denuncia in caso di violazione dei loro diritti. Ciò riafferma l'obbligo di denunciare un atto grave come l'abuso sessuale, anche se si tratta di un sospetto.

Alla luce di queste note, si comprende che l'abuso sessuale è un problema serio, con una storia ampia, che permea discussioni politiche, valori culturali (che cambiano a seconda delle regioni del mondo e con il momento storico), e per questioni di salute fisica, psicologica e sociale, che possono compromettere seriamente la vita di molti bambini e adolescenti.

\section{CONSIDERAZIONI FINALI}

Questo articolo mirava ad affrontare gli aspetti storici dell'abuso sessuale, passando attraverso la legislazione brasiliana ed esplorando le complessità della materia anche in relazione alla definizione e alla nomenclatura.

Si è visto che i bambini e gli adolescenti non sono sempre stati considerati come esseri umani che hanno bisogno di rispetto e cura specifici e che, nel corso della storia, vari tipi di violenza sono stati commessi contro questo pubblico senza punizione per gli aggressori, nemmeno l'attenzione delle autorità pubbliche per proteggere queste persone.

Di fronte a diversi casi di crudeltà e negligenza, sono state istituite leggi a favore di bambini e adolescenti e, in Brasile, I'ECA segna l'istituzione di diversi diritti, prevedendo la protezione dei minori e richiedendo agli adulti di essere responsabili della cura di loro.

Pertanto, l'abuso sessuale è uno dei fenomeni che ha attirato l'attenzione dello Stato e, attualmente, sono state stabilite misure per proteggersi da tale violenza. Gli studi dimostrano i danni biopsicosociali che questa aggressione può causare alla vittima e alla sua famiglia, nonché i meccanismi che comportano la pratica dell'abuso

RC: 98978

Disponibile in: https://www.nucleodoconhecimento.com.br/psicologia-it/aspetti-storici 
sessuale, consentendo di adottare strategie di prevenzione e di identificare e punire aggressori di diversi profili.

Tuttavia, è noto che la popolazione generale manca ancora di conoscenza dell'argomento, compresi i familiari e le istituzioni responsabili della protezione dei bambini e degli adolescenti come la scuola, per esempio. Inoltre, la mancanza di chiarimenti su questo fenomeno è un fattore che conferma che questa violenza continua a riprodursi. C'è, poi, la necessità di ulteriori ricerche e interventi che forniscano alla popolazione sempre più informazioni sul tema, sensibilizzando sull'impegno di tutti a favore della garanzia dei diritti dei bambini e degli adolescenti, potenziando così la lotta agli abusi sessuali.

\section{RIFERIMENTI}

ADED et al. Abuso sexual em crianças e adolescentes: revisão de 100 anos de literatura. Revista de Psiquiatria Clínica 33 (4); 204-213, 2006. [online]. Disponível em: <http://www.hcnet.usp.br/ipq/revista/vol33/n4/204.html>. Acesso em: 27 de mai. de 2015.

ARAÚJO, M. de F. Violência e Abuso Sexual na Família. Psicologia em Estudo, Maringá, v. 7, n. 2, p. 3-11, jul./dez. 2002. [online]. Disponível em: $<$ http://www.scielo.br/scielo.php?pid=S1413-

73722002000200002\&script=sci_arttext>. Acesso em: 09 de set. de 2015.

AZAMBUJA, M. R. F. de. Violência sexual intrafamiliar: é possível proteger a criança? Revista Virtual Textos \& Contextos, nํ5, nov. 2006. [online]. Disponível em: <http://revistaseletronicas.pucrs.br/ojs/index.php/fass/article/view/1022>. Acesso em 09 de set. de 2015.

BRASIL. Ministério da Saúde. Notificação de maus-tratos contra crianças e adolescentes pelos profissionais de saúde. Secretaria de Assistência à Saúde. Série A. Normas e Manuais Técnicos; n. 167. Brasília-DF, 2002. [online]. Disponível em:

RC: 98978

Disponibile in: https://www.nucleodoconhecimento.com.br/psicologia-it/aspetti-storici 
$<$ http://bvsms.saude.gov.br/bvs/publicacoes/notificacao_maustratos_criancas_adoles centes.pdf $>$. Acesso em: 27 de mai. de 2015.

Site: Conselho Nacional de Saúde. 18 de maio - Dia nacional de combate ao abuso e exploração sexual de crianças e adolescentes. s/d. Disponível em $<$ http://conselho.saude.gov.br/ultimas_noticias/2009/15_mai_caesca.htm>. Acesso em: 06 de jun. de 2016.

Constituição (1988). Constituição da República Federativa do Brasil: promulgada em 5 de outubro de 1988. [online]. Disponível em: <http://www.planalto.gov.br/ccivil_03/Constituicao/Constituicao.htm>. Acesso em: 07 de out. de 2016.

. Estatuto da Criança e Adolescente. Lei 8.069, de 13 de julho de 1990. Brasília, 1990. [online]. Disponível em: <http://www.planalto.gov.br/ccivil_03/LEIS/L8069.htm>. Acesso em 09 de set. de 2015.

. Superior Tribunal de Justiça. Súmula 593. O crime de estupro de vulnerável se configura com a conjunção carnal ou prática de ato libidinoso com menor de 14 anos, sendo irrelevante eventual consentimento da vítima para a prática do ato, sua experiência sexual anterior ou existência de relacionamento amoroso com o agente. Terceira Seção. Julgado em 25/10/2017. DJe 06/11/2017. Disponível em <https://www.stj.jus.br/sites/portalp/Inicio>. Acesso em: 14 de abr. de 2021.

BRINO, R. F.; WILLIAMS, L. C. Capacitação do educador acerca do abuso sexual infantile. Interação em Psicologia, 7(02), p. 1-10, 2003b. [online]. Disponível em:<file:///C:/Users/Usuario/Downloads/3218-6348-1-PB\%20(1).pdf>. Acesso em 13 de abr. de 2016.

Prevenção primária, secundária e terciária do abuso sexual infantil. In:Sobre o comportamento e cognição- Expondo a variabilidade. Org. GUILHARDI, H. J.; AGUIRRE, N. C. de. p.174-181, 2005.

RC: 98978

Disponibile in: https://www.nucleodoconhecimento.com.br/psicologia-it/aspetti-storici 
DECLARAÇÃO UNIVERSAL DOS DIREITOS HUMANOS, Adotada e proclamada pela resolução 217 A (III) da Assembléia Geral das Nações Unidas em 10 de dezembro de 1948. Representação da UNESCO no Brasil. Brasília, 1998. [online] Disponível em: <http://unesdoc.unesco.org/images/0013/001394/139423por.pdf>. Acesso em: 05 de jun. de 2016.

FALEIROS, E. T. S; CAMPOS, J. de O. C. Repensando os conceitos de violência, abuso e exploração sexual de crianças e de adolescentes. CECRIA / MJ-SEDH-DCA / FBB / UNICEF. Brasília, 2000. [online] Disponível em: $<$ http://escca.luizaugustopassos.com.br/wp-

content/uploads/2011/02/livro_repensando_os_conceitos_eva_publicacoeshttpwww.mpes_.gov_.branexoscentros_apoioarquivos1.pdf1.pdf>. Acesso em: 02 de jun. de 2016.

GUERRA, A. M. A. et al. Crianças e adolescentes têm direitos: conheça o Sistema de Garantia dos Direitos e saiba como participar. Org. GRACIANI, M. S. S. et al. 1a ed. São Paulo: CONDECA: Manufatura de ideias, 2013.

HABIGZANG, L. F. et al. Abuso sexual infantil e dinâmica familiar: aspectos observados em processos jurídicos. Psicologia: Teoria e Pesquisa, Brasília, v. 21, n.3, p.341-348, 2005.

Disponível

em:<http://www.scielo.br/scielo.php?script=sci_arttext\&pid=S0102-

$37722005000300011 \& \mathrm{lng}=\mathrm{en} \& \mathrm{nrm}=\mathrm{iso}>$. Acesso em: 07 de set. de 2016.

HABIGZANG, L. F.; RAMOS, M. da S.; KOLLER, S. H. A Revelação de Abuso Sexual: As Medidas Adotadas pela Rede de Apoio. Psicologia: Teoria e Pesquisa Out-Dez 2011, Vol. 27 n. 4, pp. 467-473. [online]. Disponível em: <http://www.scielo.br/pdf/ptp/v27n4/10.pdf>. Acesso em: 09 de set. de 2015.

HARMS, M. (org.) Vade Mecum RT. [Equipe RT]. 12 ed. revista, atualizada e ampliada. São Paulo: Editora Revista dos Tribunais, 2016. ISBN 978-85-203-6714-8.

RC: 98978

Disponibile in: https://www.nucleodoconhecimento.com.br/psicologia-it/aspetti-storici 
INOUE, S. R. V.; RISTUM, M. Violência sexual: caracterização e análise de casos revelados na escola. Estudos de Psicologia (Campinas) [online]. 2008, vol.25, n.1, pp. 11-21. ISSN 1982-0275. Disponível em: <http://www.scielo.br/scielo.php?pid=S0103166X2008000100002\&script=sci_abstract\&tlng=pt>. Acesso em: 27 de mai. de 2015.

LEMOS, F. C. S. O Estatuto da Criança e do Adolescente no Brasil atual. Revista Psicologia Política, São Paulo, v. 8, n. 15, p. 93-106, jun. 2008. Disponível em <http://pepsic.bvsalud.org/scielo.php?script=sci_arttext\&pid=S1519549X2008000100007\&lng=pt\&nrm=iso >. Acesso em:08 de jun. de 2016.

MINAYO, M. C. S. O significado social e para a saúde da violência contra crianças e adolescentes. In:WESTPHAL, M. F. (org). Violência e criança. São Paulo: Edusp, 2002.

OLIVEIRA, I. S. de. Trajetória Histórica do Abuso Sexual Contra Criança e Adolescente. UniCEUB: Brasília. 2006. Professor-orientador: Dr. Maurício Neubern.

PADILHA, M. da G. S. Abuso sexual contra crianças e adolescentes: considerações sobre os fatores antecedentes e sua importância na prevenção. In: GUILHARDI, H. J.; AGUIRRE, N. C. (Orgs.) Sobre Comportamento e Cognição: Expondo a variabilidade. Santo André: ESETec, 2002, v.10, p. 209 a 220.

PAPALIA, D.; OLDS, S.W.; FELDMAN, R. D. Desenvolvimento Humano. 8 ed.Porto Alegre: Artmed, 2006.

PRADO, M. do C. de A.; CARNEIRO, T. F. Abuso sexual e traumatismo psíquico. Interações, São Paulo, v. 10, n. 20, p. 11-34, dez. 2005. [online] Disponível em:<http://pepsic.bvsalud.org/scielo.php?script=sci_arttext\&pid=S1413$29072005000200002 \& \operatorname{lng}=p t \& n r m=i s o>$. Acesso em: 23 de abr. de 2016.

SANTOS. B. R. dos; IPPOLITO, R - Childhood Brasil. O papel da escola no enfrentamento da violência sexual. In: LAVARELLO, F. (Coord). A defesa de

RC: 98978

Disponibile in: https://www.nucleodoconhecimento.com.br/psicologia-it/aspetti-storici 
crianças e adolescentes vítimas de violências sexuais. São Paulo: Cromosete, 2009, p. $125-130$.

SANTOS, V. A. dos; COSTA, L. F.; GRANJEIRO, I. A. C. L. Intervenção no abuso sexual intrafamiliar: ingerência invasiva ou proteção devida? Psico, Porto Alegre, PUCRS, v. 40, n. 4, pp. 516-524, out./dez. 2009. [online]. Disponível em: <http://revistaseletronicas.pucrs.br/ojs/index.php/revistapsico/article/view/4009>. Acesso em: 09 de set. de 2015.

SERAFIM, A. de P. et al. Dados demográficos, psicológicos e comportamentais de crianças e adolescentes vítimas de abuso sexual. Revista de Psiquiatria Clínica, São Paulo, v. 38, n. 4, p. 143-147, 2011. [online]. Disponível em: <http://www.scielo.br/scielo.php?script=sci_arttext\&pid=S0101 60832011000400006>. Acesso em: 09 de set. de 2015.

UNICEF. Situação mundial da infância (edição especial): celebrando 20 anos da Convenção sobre os Direitos da Criança. UNICEF, 2009. [online] Disponível em: $<$ http://www.unicef.org/brazil/pt/sowc_20anosCDC.pdf>. Acesso em: 03 de jun. de 2016.

VEGA, L. B. da S.; PALUDO, S. dos S. Exploração sexual e rede de proteção na perspectiva da vítima. Arquivos Brasileiros de Psicologia, Rio de Janeiro, v. 67, n. 2, p. 47-60, $2015 . \quad$ Disponível em $<$ http://pepsic.bvsalud.org/scielo.php?script=sci_arttext\&pid=S1809$52672015000200005 \&$ Ing=pt\&nrm=iso >. Acesso em: 29 de maio de 2016.

WAISELFISZ, J. J. Atendimento por Violência No SUS. In: Mapa da Violência 2012: Crianças e Adolescentes do Brasil. 1 ed. Rio de Janeiro: CEBELA, 2012. p.62-73.

WILLIAMS, L. C. A. Abuso sexual infantil. In: GUILHARDI, H. J.; AGUIRRE, N. C. (Orgs.). Sobre Comportamento e Cognição: Expondo a variabilidade. Santo André: ESETec, 2002, v.10, p.155-164.

RC: 98978

Disponibile in: https://www.nucleodoconhecimento.com.br/psicologia-it/aspetti-storici 


\section{APPENDICE - NOTA A PIÈ DI PAGINA DI RIFERIMENTO}

3. Dati ottenuti in https://agenciapatriciagalvao.org.br/violencia/violencia-sexual/trescriancas-ou-adolescentes-sao-abusadas-sexualmente-no-brasil-a-cada-hora/

4. Sito web del portale Brasile: < http://www.brasil.gov.br/cidadania-ejustica/2015/07/em-1927-o-brasil-ganhou-oprimeiro-codigo-de-menores> .

5. Sito web di UDHR: < http://www.dudh.org.br/declaracao/> .

6. Sito Web CRAMI: <http://www.cramicampinas.org.br

7. Sito web dell'UNICEF: <http: www.unicef.org/brazil/pt/activities_10790.htm="'>.</http:>

Inviato: Maggio 2021.

Approvato: Settembre 2021.

RC: 98978

Disponibile in: https://www.nucleodoconhecimento.com.br/psicologia-it/aspetti-storici 